\title{
Argentina: efectos del programa Asignación Universal por Hijo en el comportamiento laboral de los adultos
}

\author{
Roxana Maurizio y Gustavo Vázquez
}

RESUMEN

En 2009, la Argentina impulsó el programa Asignación Universal por Hijo para Protección Social, plan de transferencias monetarias para hogares con niños. Se amplió así a los padres desempleados u ocupados en el sector informal la cobertura del programa de asignaciones familiares contributivas existente. En este artículo se evalúan los efectos a corto plazo del programa en la participación laboral de los adultos y en la generación de ingresos. Se utilizó el estimador de diferencias en diferencias en conjunto con técnicas de emparejamiento por índices de propensión (propencity score matching). Los resultados sugieren que, en el primer año de operación, el programa no ocasionó desincentivos importantes, pues no desalentó el trabajo de los adultos ni originó una reducción del número de horas trabajadas. Hallazgos muy relevantes en el contexto latinoamericano, donde las transferencias monetarias de este tipo se han convertido en un componente fundamental de los sistemas de protección social.

PALABRAS CLAVE

CLASIFICACIÓN JEL

AUTORES
Seguridad social, bienestar de la infancia, hogares, prestación por hijos a cargo, programas de acción, ingresos familiares, evaluación, Argentina

J22, I38, J08
Roxana Maurizio es investigadora docente de la Universidad Nacional de General Sarmiento y del Consejo Nacional de Investigaciones Científicas y Técnicas (CONICET), Argentina. roxanadmaurizio@gmail.com Gustavo Vázquez es investigador docente de la Universidad Nacional de General Sarmiento, Argentina. gmvazque@ungs.edu.ar 


\section{I}

\section{Introducción}

En 2009, en la Argentina se puso en marcha un importante programa de transferencias monetarias para niños y adolescentes, denominado Asignación Universal por Hijo para Protección Social, que extendió la cobertura de las asignaciones familiares contributivas a nuevos segmentos de la población.

La Asignación Universal por Hijo consiste en una transferencia monetaria que se paga mensualmente a un padre, tutor o familiar (hasta el tercer grado de consanguinidad) por cada niño menor de 18 años que esté a su cargo. En el caso de los niños con discapacidades, la edad límite no se aplica. Se trata de una transferencia monetaria semicondicionada: el $80 \%$ de su valor se paga mensualmente al beneficiario y el $20 \%$ restante se deposita en una cuenta de ahorro a su nombre. El beneficiario puede retirar esta última suma cuando demuestre que sus hijos asisten a la escuela y se realizan controles médicos. Los beneficiarios no pueden solicitar otros beneficios sociales otorgados por el gobierno nacional, provincial o municipal o por la Ciudad Autónoma de Buenos Aires; además, todos los programas anteriores orientados a grupos similares se eliminaron progresivamente.

En varios estudios se efectuaron evaluaciones previas que simulaban el efecto de la asignación en los indicadores de igualdad, pobreza y extrema pobreza ${ }^{1}$. En todos los casos, se llegó a la conclusión de que, una vez que su cobertura alcanzara a toda la población destinataria, la Asignación Universal por Hijo reduciría significativamente la indigencia $-\mathrm{y}$ en menor medida, la pobreza- y tendría a la vez una incidencia positiva en la desigualdad. Sin embargo, en estos estudios no se tiene en cuenta la posible repercusión de estas transferencias en la decisión de trabajar de los adultos y en el número de horas que dedican al trabajo.

En el presente artículo se propone cerrar esta brecha, realizando una evaluación ex post de la Asignación

Los autores agradecen profundamente las valiosas opiniones y sugerencias de Fabio Bertranou, Rafael Rofman y Luis Beccaria, como también los útiles comentarios de los dos revisores anónimos.

1 Entre estos, se encuentran los de Roca (2010); Agis, Cañete y Panigo (2010); Gasparini y Cruces (2010); OIT (2010), y Bertranou y Maurizio (2012).
Universal por Hijo. Mediante la aplicación de una estrategia econométrica no experimental, se evalúa el efecto a corto plazo de la asignación en las decisiones relativas a la participación económica, el empleo, el desempleo, el número de horas trabajadas y la generación de ingresos. De esta manera, el estudio contribuye a enriquecer la escasa pero creciente literatura sobre el efecto de las transferencias monetarias en el comportamiento laboral de los adultos en los países en desarrollo.

Considerando los resultados obtenidos, no es posible concluir que el programa haya generado desincentivos al trabajo entre los adultos miembros de los hogares beneficiarios en los años 2009 y 2010 , en el sentido de alentarlos a dejar la fuerza laboral o a reducir el número de horas de trabajo.

Sin embargo, cabe aclarar al menos cuatro puntos en relación con estos resultados. Primero, a diferencia de otros casos en los que se realizan análisis de impactos como parte de una evaluación de este tipo de programas, en la Argentina no se llevó a cabo ninguna encuesta específicamente diseñada para este propósito. Segundo, en la encuesta de hogares utilizada, los beneficiarios de la Asignación Universal por Hijo solo se identifican indirectamente. Tercero, podrían surgir diferencias en las características no observadas del grupo de tratamiento y el grupo de control, aunque la metodología empleada se orienta a reducir la probabilidad de este problema. Por último, este análisis cubre un período de tiempo relativamente corto y los resultados pueden cambiar a lo largo del horizonte temporal, sobre todo en lo que respecta a los efectos del programa en la participación laboral.

En la sección II de este artículo se describen brevemente las principales características del programa. En la sección III se presenta el marco teórico y un análisis de la evidencia empírica de América Latina. Las fuentes de información utilizadas se detallan en la sección IV, y en la quinta sección se especifica la estrategia econométrica. La sección VI contiene algunas estadísticas descriptivas, y en la sección VII se discuten los efectos en el funcionamiento del mercado laboral. Por último, en la sección VIII se ofrecen algunos comentarios finales. 


\section{II}

\section{Breve descripción del programa}

Dada la gran incidencia de la pobreza entre los niños y adolescentes en comparación con otros grupos etarios, desde mediados de la década de 1990 los países de América Latina han iniciado y ampliado programas de transferencias monetarias no contributivas (transferencias monetarias condicionadas) dirigidos a este segmento de la población. Gradualmente, estos programas se convirtieron en importantes mecanismos de las políticas sociales y estrategias regionales de reducción de la pobreza.

En noviembre de 2009, el Gobierno de la Argentina inició la aplicación de un importante programa de transferencias monetarias para los hogares con niños, denominado Asignación Universal por Hijo para Protección Social, mediante el cual se extendió la cobertura del plan de asignaciones familiares contributivas existente a los hijos de:

i) los trabajadores no registrados en el sistema de seguridad social (trabajadores informales) y los empleados domésticos con un ingreso laboral inferior al salario mínimo;

ii) los monotributistas sociales ${ }^{2}$;

iii) los desempleados sin seguro de desempleo, y

iv) los trabajadores económicamente inactivos que no perciben pensiones.

Se excluyeron de esta asignación dos grupos de trabajadores, a pesar de que no reciben ningún beneficio contributivo: los trabajadores domésticos cuyos ingresos exceden el salario mínimo y los trabajadores registrados en el esquema de monotributo, excepto aquellos que pertenecen al monotributo social.

La Asignación Universal por Hijo es una transferencia monetaria que se paga mensualmente a un padre, tutor o familiar (hasta el tercer grado de consanguinidad) por cada niño menor de 18 años de edad que esté a su cargo. Este límite de edad no se aplica en el caso de los niños con discapacidades. Los niños deben ser ciudadanos argentinos o haber residido en el país durante por lo menos tres años. Tanto el niño como los padres deben

\footnotetext{
${ }^{2}$ El monotributo es un régimen tributario simplificado bajo el cual el trabajador paga una única suma fija (cuyo valor depende del ingreso declarado), formada por un componente previsional y un componente impositivo. El monotributo social es una categoría tributaria para personas en situación de vulnerabilidad social que forman parte de cooperativas de trabajo o proyectos de producción de hasta tres personas, y cuyos ingresos sean inferiores a determinado nivel.
}

tener documento nacional de identidad. Si los padres comparten la custodia, las madres tienen prioridad para recibir el beneficio, que consiste en una suma fija por cada niño a cargo hasta un máximo de cinco niños. Su valor inicial era de 180 pesos (47 dólares) por niño y 720 pesos por cada niño con discapacidad (cuatro veces el monto normal). Posteriormente, el valor se actualizó teniendo en cuenta el deterioro de su poder adquisitivo a causa de la inflación.

Actualmente, la Asignación Universal por Hijo cubre al 30\% de los niños (3,5 millones) y el $15 \%$ de los hogares (1,8 millones) de la Argentina. El gasto del gobierno en el programa representa aproximadamente el $0,8 \%$ del producto interno bruto (PIB), lo que lo convierte en uno de los programas de mayor envergadura de la región.

La recepción de otro tipo de beneficio social es incompatible con esta asignación, de modo que se eliminaron los programas anteriores orientados a grupos similares. En particular, la nueva política reemplazó al Plan Jefes y Jefas de Hogar Desocupados (PJJHD) y al Programa Familias por la Inclusión Social, los dos programas de transferencias monetarias condicionadas más importantes de la década de 2000. Como resultado, en sus etapas iniciales, la Asignación Universal por Hijo dio cobertura a un gran número de personas que habían sido beneficiarias de esos dos programas.

La Asignación Universal por Hijo es un programa de transferencias monetarias semicondicionadas: el $80 \%$ de su valor se paga mensualmente al beneficiario y el $20 \%$ restante se deposita en una cuenta de ahorro a su nombre. Esta última suma puede ser retirada una vez que el beneficiario demuestre haber completado el plan de vacunación y los controles sanitarios pertinentes, en el caso de los niños menores de 5 años, o haya presentado un certificado de finalización del ciclo lectivo en el caso de los niños en edad escolar ${ }^{3}$. Por lo tanto,

\footnotetext{
3 Las disposiciones del programa establecen que se suspenderá el pago mensual de la asignación si no se presentan debidamente los certificados y que no se podrá retirar el depósito del $20 \%$ hasta que se regularice la situación. En ciertos casos, los padres no cumplían con las condiciones debido a la ausencia de centros de vacunación cercanos o porque no conseguían concertar una cita con el médico que certificaba los controles de salud (Pautassi, Arcidiácono y Straschnoy, 2013). Por esta razón, los controles sobre el cumplimiento de las condiciones no eran exhaustivos en las primeras etapas del programa, pero se hicieron más rigurosos con el tiempo.
} 
las condicionalidades estipuladas en la Asignación Universal por Hijo son similares a las de la mayoría de los programas de transferencias monetarias establecidos en América Latina.

Sin embargo, si bien la reducción de la pobreza y la pobreza extrema es uno de sus objetivos, la Asignación Universal por Hijo no es un programa ad hoc diseñado para aliviar la situación de las familias con niños socialmente vulnerables, como es el caso del programa Bolsa Familia, del Brasil, u Oportunidades, de México. Como se mencionó anteriormente, es una extensión del ya existente programa de asignaciones contributivas por hijo, que cubre a los hijos de los trabajadores formales, a los desempleados con seguro de desempleo y a los pensionados. De hecho, la suma percibida por menor a cargo es la misma en ambos sistemas.

Este punto es importante porque, a diferencia de esos programas de transferencias monetarias condicionadas, en este caso las restricciones impuestas no se relacionan directamente con los ingresos familiares, sino con la situación laboral de los adultos encargados de los niños $\mathrm{y}$ con sus ingresos laborales (si trabajan en empleos informales). Sin embargo, las dificultades que acarrea supervisar los ingresos laborales de los trabajadores no registrados en la seguridad social debilitan el cumplimiento de tales restricciones.

\section{III}

\section{Marco teórico y evidencia empírica}

\section{Marco teórico}

Existe en la actualidad un intenso debate acerca de las consecuencias que pueden tener las transferencias monetarias a los hogares en el comportamiento laboral de los adultos. Esas consecuencias se relacionan con la percepción de nuevos ingresos no laborales, por una parte, y con el cumplimiento de las condicionalidades del programa, por otra.

En la teoría neoclásica sobre la oferta individual de trabajo se sostiene que este tipo de ingresos no laborales producen un efecto puro en el ingreso de los hogares, lo que origina un aumento de la demanda de bienes normales. Si el ocio es un bien normal, la oferta de trabajo descenderá, lo que puede conducir a salidas del mercado laboral (solución de esquina) o a una reducción del número de horas trabajadas (solución interior).

No obstante, podría argumentarse que el efecto real de las transferencias monetarias dependerá de su magnitud. Otros factores también podrían afectar a la decisión de permanecer o abandonar la fuerza laboral en respuesta a esos beneficios, como por ejemplo, ciertas características del empleo diferentes de la remuneración (condiciones de trabajo, distancia de traslado o número de horas trabajadas) o las demandas de cuidado y tareas hogareñas.

Por otra parte, puede argumentarse que si los montos de las transferencias son lo suficientemente elevados como para desalentar la participación laboral, también pudieran producir el efecto opuesto. En otras palabras, este beneficio podría permitir que los hogares superen las barreras de ingreso a ciertas actividades productivas o empresariales o que tomen ciertas decisiones económicas que de otro modo serían imposibles (Medeiros, Britto y Veras Soares, 2008; Teixeira, 2010).

En el modelo más complejo de oferta laboral familiar (Killingsworth, 1983), las decisiones relativas a la distribución del tiempo se vinculan a las decisiones de otros miembros del hogar. De ese modo, sería posible introducir un segundo canal por cuyo intermedio las transferencias podrían originar cambios en el comportamiento laboral de los adultos, es decir, ocasionar los efectos vinculados al cumplimiento de las condiciones del programa. Al estar ligado a la concurrencia a la escuela, el beneficio reduce el costo de oportunidad de estudiar, lo que podría derivar en un descenso de la demanda de bienes sustitutivos del estudio y en un incremento de la demanda de bienes complementarios del estudio. Si el trabajo es un sustituto de los estudios, se reducirá la oferta de trabajo de los menores. Sin embargo, si el trabajo y los estudios no son sustitutos perfectos, el efecto en la oferta de trabajo de los menores podría ser ambiguo (Ravallion y Wodon, 2000).

$\mathrm{Al}$ respecto surgen dos cuestiones relevantes sobre la oferta de trabajo de los adultos. Una se refiere a cómo reaccionaría la oferta de mano de obra de otros miembros del hogar ante una reducción de la oferta de trabajo de los niños. Esta reacción podría compensar parcialmente el efecto de las transferencias en los ingresos del hogar, de modo que el potencial desincentivo de los adultos 
sería bastante exiguo. Skoufias y Parker (2001) señalan que la repercusión de estas transferencias variará de un hogar a otro dependiendo de sus circunstancias particulares. Por ejemplo, en ciertos tipos de hogares, el monto de la transferencia podría ser inferior a la pérdida del ingreso causada por la reducción de la oferta laboral de los menores una vez que comienzan a concurrir a la escuela, lo que desalentaría la afiliación al programa.

El segundo punto se relaciona con que el cumplimiento de las condiciones podría por sí mismo alterar la asignación del tiempo de los adultos: si la asistencia a la escuela reduce el tiempo dedicado al cuidado de los niños, podría incrementarse el tiempo disponible para trabajar; por el contrario, el tiempo necesario para garantizar la concurrencia al colegio y los controles médicos podría reducir las horas destinadas al trabajo (Parker y Skoufias, 2000).

Por consiguiente, la distribución del tiempo y las tareas dentro de los hogares son otro aspecto central de este tipo de transferencias, y estos factores se vuelven más relevantes desde la perspectiva de género. Como señala Gammage (2010), al analizar los resultados de estos programas deben tomarse en cuenta los efectos potenciales que tienen en el tiempo que las mujeres destinan al trabajo remunerado y al trabajo no remunerado, dado que la responsabilidad de las tareas derivadas del cumplimiento de los requisitos recae generalmente en ellas.

Por último, el hecho de que estos programas tengan una puerta de salida vinculada a la mejora de las condiciones económicas de los hogares podría desalentar la participación en el mercado laboral, dado que esto afectaría a la elegibilidad para continuar en el programa. En el caso de la Asignación Universal por Hijo, acceder al programa y permanecer en él no depende de los ingresos familiares, sino de los ingresos laborales de los adultos encargados de los niños. Sin embargo, como ya se mencionó, supervisar el cumplimiento de este requisito es bastante difícil en un contexto de informalidad laboral, lo que podría disminuir la ocurrencia de comportamientos tendientes a reducir la oferta de trabajo. En el caso de los desempleados o las personas económicamente inactivas, la Asignación Universal por Hijo no ocasiona ningún desincentivo explícito al trabajo, puesto que un trabajo formal les daría acceso a una asignación por hijo contributiva, mientras que un trabajo informal les permitiría seguir recibiendo los beneficios del programa. Además, el beneficio consiste en una suma fija por hijo y no depende del nivel de ingresos laborales. Todas estas características particulares de la Asignación Universal por Hijo podrían disminuir los posibles efectos de las transferencias en las decisiones relativas al trabajo.

\section{Evidencia empírica para los países de América Latina}

Los efectos de los programas de transferencias monetarias condicionadas en el comportamiento laboral de los adultos en América Latina se están analizando en un número creciente de estudios. Sin embargo, la evidencia empírica no es concluyente.

Los resultados obtenidos por Ferro y Nicollela (2007) en el caso del programa brasileño Bolsa Familia sugieren que este no tuvo un efecto disuasivo en la oferta de mano de obra, aunque sí en el número de horas trabajadas, pero el efecto agregado parece haber sido bastante pequeño. En particular, si bien las madres beneficiarias que viven en zonas urbanas trabajan 1,5 horas por semana más que las no beneficiarias, las madres y los padres de zonas rurales presentan un comportamiento opuesto. La reducción del número de horas trabajadas podría deberse a que destinan más tiempo al cumplimiento de las condiciones del programa o a que previamente los niños se encargaban del trabajo doméstico. El potencial efecto de las transferencias en el ingreso también puede haber ejercido influencia. En un estudio posterior realizado por Ferro, Kassouf y Levison (2010) se obtuvieron resultados similares: el programa originó un aumento de la participación económica de las madres y los padres en las zonas urbanas, pero no tuvo repercusiones importantes en las zonas rurales.

Foguel y Paes de Barros (2010) encontraron que el programa tenía efectos nulos en la participación económica femenina y una pequeña repercusión en la masculina. En cuanto al número de horas trabajadas, observaron un efecto negativo de escasa magnitud en el caso de las mujeres y ningún efecto significativo en el caso de los hombres.

Según Medeiros, Britto y Veras Soares (2008), las mujeres jefas de hogar que recibían la asignación tenían menos tendencia a participar en el mercado laboral que las no beneficiarias. En los otros grupos no se observaron efectos significativos. Los resultados obtenidos por Teixeira (2010) sugieren también un efecto medio nulo del programa Bolsa Familia en la probabilidad de trabajar y una reducción muy pequeña del número de horas trabajadas por los adultos. La elasticidad de la respuesta es mayor entre las mujeres y los trabajadores informales, y se incrementa en función del tamaño del beneficio.

En cambio, Soares, Ribas y Osório (2007) observaron un aumento en las tasas de participación de hombres y mujeres vinculado al programa Bolsa Familia, con mayor variación entre las mujeres. En el mismo sentido, Tavares (2008) descubrió que la probabilidad de trabajar 
se acrecienta alrededor de un $6 \%$ en el caso de las madres beneficiarias, mientras que el número de horas que trabajan por semana se incrementa un $2 \%$.

En el caso de México, Parker y Skoufias (2000) observaron que el programa Progresa (actualmente, programa Oportunidades) no tiene efectos disuasivos en las decisiones laborales de los adultos. Un análisis más detallado de la distribución del tiempo de las mujeres revela que el programa produjo un aumento del número de horas destinadas a cumplir con las condicionalidades de este. Por otra parte, no tiene efectos significativos en el número de horas dedicadas al esparcimiento, tanto en el caso de los hombres como en el de las mujeres. Los resultados obtenidos por Skoufias y Di Maro (2008) confirman que el programa mexicano no causa efectos disuasivos.

Un esquema similar se encuentra en el Uruguay, donde la introducción del Plan de Atención Nacional a la Emergencia Social (PANES) no originó cambios en la oferta de mano de obra ni en el número de horas trabajadas (Amarante, Ferrando y Vigorito, 2011).

Soares, Ribas e Hirata (2008), al analizar el impacto del programa Tekoporã, descubrieron que tenía un efecto negativo en la oferta de mano de obra masculina, e incluso mayor en las zonas moderadamente pobres y en las rurales. Sin embargo, cuando se excluye a los trabajadores temporales, este efecto se observa solo en las zonas moderadamente pobres. En el caso de las mujeres y de la población en su conjunto, se observaron efectos no significativos.

Galasso (2006) analizó los efectos del programa Chile Solidario durante los primeros dos años de funcionamiento y encontró que, si bien el acceso a este beneficio originó una mayor participación en los programas de empleo - lo que podría mejorar la empleabilidad en

\section{IV}

\section{Datos utilizados}

Los datos utilizados en este artículo provienen de la Encuesta Permanente de Hogares (EPH) de la Argentina, realizada por el Instituto Nacional de Estadística y Censos (INDEC), que cubre 31 aglomerados urbanos y recoge información especialmente sobre variables del mercado laboral.

Si bien la EPH no es longitudinal y no incluye preguntas retrospectivas, su muestra de panel rotatorio el mediano plazo—, la proporción global de miembros empleados en los hogares beneficiarios no parece haber aumentado en el corto plazo. Solo en las zonas rurales se observó un incremento de la tasa de participación en la fuerza de trabajo. En todo caso, la autora destaca que el horizonte de corto plazo del análisis podría no ser suficiente para observar cualquier repercusión en esas dimensiones.

Alzúa, Cruces y Ripani (2010) condujeron una evaluación comparativa de los programas de transferencias en Honduras, México y Nicaragua. Nuevamente hallaron que las decisiones referentes a la participación laboral y las horas de trabajo no se ven afectadas por estas transferencias, excepto en Nicaragua, donde se redujo el número de horas trabajadas a nivel de los hogares, en especial en los casos de jefatura femenina. De hecho, los autores encontraron que el programa Progresa tenía efectos positivos en los salarios por hora de los hombres y en el ingreso laboral total de los hogares beneficiarios, lo que sugiere la presencia de efectos indirectos en las condiciones del mercado de trabajo local.

Por último, Garganta y Gasparini (2012) evaluaron los efectos de la Asignación Universal por Hijo en las transiciones entre la formalidad y la informalidad, y concluyeron que el programa desalentó de manera significativa la formalización de los beneficiarios, pero no hallaron evidencia de incentivos para que los trabajadores formales se convirtieran en informales.

Por lo tanto, es posible concluir que la evaluación de los impactos de la Asignación Universal por Hijo es una cuestión empírica. Sin embargo, la evidencia más reciente para los países de América Latina sugiere, al mismo tiempo, que las transferencias monetarias no tienen efectos disuasivos importantes en la inserción laboral de los beneficiarios adultos. permite obtener datos de flujo, es decir, que se entrevista a un hogar seleccionado en cuatro momentos u ondas: el hogar aparece en la muestra durante dos trimestres consecutivos, seguidos de una pausa de dos trimestres, y aparece nuevamente en dos trimestres consecutivos un año después. Comparando la situación de un individuo en determinada onda con la del mismo individuo en otra onda, es posible determinar si la persona ha 
experimentado cambios en diversas variables, incluidas las ocupacionales y demográficas.

En particular, en este estudio se emplean datos de panel anuales construidos para el período comprendido entre el primer trimestre de 2009 y el tercer trimestre de 2010, con el fin de incluir información previa y posterior a la puesta en marcha de la Asignación Universal por Hijo en noviembre de $2009^{4}$. Para garantizar el mayor número de observaciones disponibles, se trabajó con una única base conteniendo los datos de estos tres paneles anuales.

${ }^{4}$ Los datos del cuarto trimestre de 2009 se excluyeron debido a que el programa se lanzó en noviembre de ese año y ese período ya contiene información sobre los beneficiarios del programa.

\section{Enfoque y metodología}

\section{Especificación econométrica}

Con el propósito de evaluar los efectos de la Asignación Universal por Hijo, se utilizó un método no experimental basado en la aplicación de técnicas de emparejamiento para definir un grupo de control, lo que permite estimar cuál habría sido la situación de los beneficiarios si no hubieran accedido al programa. Tras definir con precisión el grupo de control y comparando las variables de resultado del grupo beneficiario con las del primero, es posible atribuir las diferencias observadas a la política particular que se estudia.

Siguiendo la terminología tradicional de este enfoque, $D$ se define como la variable que indica la recepción de la transferencia $(D=1$ si el hogar o la persona recibe la transferencia; $D=0$ si no la recibe); $Y$ es el resultado de interés (donde $Y^{1}$ es el resultado en presencia del beneficio e $Y^{0}$ en su ausencia). El impacto de la transferencia se mide por el efecto medio del tratamiento en los tratados, que depende de un índice de propensión $\mathrm{P}(\mathrm{X})$, donde $\mathrm{X}$ representa un vector de características observables:

$$
A T T(X)=E\left[Y^{1}-Y^{0} / P(X), D=1\right]
$$

donde $E[$.$] es el valor esperado de la diferencia entre los$ dos resultados, con y sin el tratamiento, en la población que recibe la transferencia $(D=1)$.

Dado que el contrafactual $E\left[Y^{0} / P(X), D=1\right]$ no refiere a una situación observable, para estimarlo se emplean técnicas de emparejamiento a partir de un índice de propensión. Teniendo en cuenta que solo es necesario identificar el efecto medio del tratamiento en los tratados, es suficiente verificar los supuestos sugeridos en Heckman, Ichimura y Todd (1997 y 1998): i) la "ignorabilidad" o independencia del tratamiento en media condicional, y ii) la condición de emparejamiento o matching. La primera condición implica que el grupo de tratados y el grupo de control se seleccionaron solo sobre la base del índice de propensión; entonces, después de dar cuenta de ello, la asignación al tratamiento es independiente de la media de los resultados; la segunda condición garantiza que por cada valor posible del índice de propensión existen casos beneficiarios y no beneficiarios.

Para estimar el parámetro asociado con el efecto medio del tratamiento en los tratados, se implementará un estimador de diferencias en diferencias (DD) sobre la base de la información disponible correspondiente a los períodos anterior y posterior a la implementación de la política, comparando los cambios temporales de la variable de resultado en el grupo beneficiario con los cambios de la misma variable en el grupo de control. La ventaja de esta estrategia reside en la posibilidad de controlar los sesgos derivados de las características no observables invariables en el tiempo. Su expresión está dada por:

$$
\begin{aligned}
& \widehat{A T T^{D D}}=\frac{1}{n^{1}} \sum_{\substack{\left.i=1 \\
n^{1}=1\right\}}}^{1} Y_{i, t_{1}}^{1}\left(X_{i, t_{1}}\right)-Y_{i, t_{0}}^{1}\left(X_{i, t_{0}}\right)- \\
& \widehat{E}\left[Y_{i, t_{1}}^{0}\left(X_{i, t_{1}}\right)-Y_{i, t_{0}}^{0}\left(X_{i, t_{0}}\right) / P\left(X_{i, t_{0}}\right), D_{i}=0\right]
\end{aligned}
$$

donde $n^{1}$ representa la cantidad de casos que reciben el beneficio, $t_{0}$ es el momento anterior a la puesta en marcha del programa y $t_{1}$ el momento posterior a su implementación.

De este modo, adaptando los supuestos i) y ii) al contexto de este estimador, se derivan las siguientes expresiones: 


$$
\begin{gathered}
E\left[Y_{t_{1}}^{0}(X)-Y_{t_{0}}^{0}(X) / P(X), D=1\right]= \\
E\left[Y_{t_{1}}^{0}(X)-Y_{t_{0}}^{0}(X) / P(X), D=0\right] \\
0<\operatorname{Pr}(D=1 / X)<1
\end{gathered}
$$

Finalmente, la atención se centrará en la estimación del parámetro del efecto medio del tratamiento en los tratados, en el soporte del índice de propensión común al grupo de beneficiarios y de control. Para estimar el contrafactual involucrado, se aplican alternativamente dos métodos de emparejamiento: el método del "vecino" más cercano ${ }^{5}$ y el de regresiones lineales locales ${ }^{6}$.

\section{Estrategias para identificar el grupo de tratamiento y el grupo de control}

Este estudio se basa en la identificación correcta de los hogares beneficiarios de la Asignación Universal por Hijo (grupo de tratamiento) y los que constituirán el grupo de control.

Lamentablemente, en la EPH no se indaga sobre este tema de manera directa, de modo que la identificación se debe efectuar indirectamente. Con el objetivo de identificar los hogares que recibían la asignación en 2010, se recurrió a la pregunta que captura la suma de transferencias monetarias otorgadas a los miembros del

\footnotetext{
5 De acuerdo con esta alternativa, la construcción del contrafactual de la variable de resultado para cada caso tratado se efectúa a partir de un promedio simple de las realizaciones de la misma variable para un subconjunto de casos pertenecientes al grupo de control, cercanos en términos de la probabilidad condicional de recibir el beneficio, dado un conjunto de atributos observables. En el presente trabajo, cada subconjunto estará compuesto por los 5 "vecinos" más cercanos. ${ }^{6}$ En este caso, cada contrafactual se compone a partir de un promedio ponderado de las realizaciones de la variable de resultado del subconjunto de casos del grupo de control asignado a cada unidad beneficiaria, en términos de la medida de proximidad comentada en la nota al pie 5. En este sentido, los pesos se construyen de forma tal de asignar mayor importancia a las unidades de comparación más cercanas al caso beneficiario respectivo, y se estiman para cada subgrupo a partir de regresiones lineales ponderadas de la variable de resultado en una constante y la diferencia entre las estimaciones de los índices de propensión con la correspondiente al caso beneficiario que se trate. Los ponderadores empleados en las regresiones involucran una función de kernel cuyos argumentos están dados por la medida de proximidad considerada, y el ancho de banda o parámetro de suavizado seleccionado (concepto análogo al número de "vecinos" considerados en el contexto de la primera alternativa). Fan (1992) analiza las propiedades de este estimador comparadas con aquellas asociadas a otros de su tipo, y concluye que es el más eficiente en términos asintóticos y en muestras finitas, a la vez que se adapta a diferentes diseños de densidad de datos.
}

hogar por el gobierno, las instituciones privadas o la Iglesia, entre otras instituciones. La pregunta incluye un amplio rango de entidades, por lo que no puede asumirse que las respuestas se relacionen exclusivamente con este programa. En consecuencia, los hogares se clasificaron en un comienzo como beneficiarios de la Asignación Universal por Hijo solo cuando los montos declarados coincidían con los valores establecidos en el programa, es decir, el monto de la transferencia se utilizó como indicador de tratamiento.

$\mathrm{Al}$ considerar la frecuencia de los valores monetarios que aparecen en esta pregunta, puede asumirse que algunos hogares declararon la suma que realmente recibían cada mes como beneficio (el $80 \%$ de la suma del beneficio), en tanto que otros declararon el monto total. Los valores de la asignación por número de hijos a cargo en el período analizado se presentan en el cuadro 1 .

CUADRO 1

Argentina: valor de la Asignación Universal por Hijo, por número de hijos, 2010 (En pesos argentinos)

\begin{tabular}{lcc}
\hline \multirow{2}{*}{$\begin{array}{l}\text { Número } \\
\text { de hijos }\end{array}$} & \multicolumn{2}{c}{ Valor de la Asignación Universal por Hijo } \\
\cline { 2 - 3 } & Total & $80 \%$ \\
\hline 1 & 180 & 144 \\
2 & 360 & 288 \\
3 & 540 & 432 \\
4 & 720 & 576 \\
5 & 900 & 720 \\
\hline
\end{tabular}

Fuente: elaboración propia sobre la base de datos de la Encuesta Permanente de Hogares (EPH) del Instituto Nacional de Estadística y Censos (INDEC).

Sin embargo, los valores cercanos a la suma del beneficio también se consideraron como Asignación Universal por Hijo teniendo en cuenta la posibilidad de errores en las declaraciones de ingresos y el hecho de que los hogares tienden a redondear los montos declarados. Para minimizar la posibilidad de una mala clasificación, la frecuencia de cada uno de estos valores en 2010 se comparó con la de 2009 (antes de la puesta en marcha del programa), con el fin de verificar que los valores considerados como Asignación Universal por Hijo no estaban presentes el año anterior a la implementación. Este procedimiento mostró con claridad cómo los valores correspondientes a las transferencias de la asignación comenzaron a aparecer mientras los pagos hechos por otros programas nacionales habían empezado a desaparecer (esto se aplica al Plan Jefes y Jefas de Hogares Desocupados (PJJHD), al Programa Familias por la Inclusión Social y al Seguro de Capacitación y Empleo), debido a que la 
Asignación Universal por Hijo no puede percibirse junto con otro tipo de beneficio social (véase el gráfico 1$)^{7}$.

Además, cuando los valores observados sugerían que más de una persona por hogar estaba recibiendo la asignación, el total del beneficio recibido por el hogar se comparó con el número de hijos. Dado que se encontraron muchos casos en que la suma del beneficio aparecía erróneamente para más de un miembro adulto, se excluyó del análisis a los hogares con más de un miembro receptor y a aquellos cuyo ingreso total de la asignación sugería la presencia de más niños que los que realmente vivían en el hogar ${ }^{8}$.

\footnotetext{
${ }^{7}$ Sobre la base del gráfico 1, parece razonable asumir que los valores tales como 140 pesos y 280 pesos también corresponden a la Asignación Universal por Hijo porque, por una parte, estos valores aparecen en $2010 \mathrm{y}$, por otra, porque los montos son muy similares a los establecidos en el programa (144 pesos y 288 pesos, respectivamente).

${ }^{8}$ Estos hogares representaban un 3\% del número total de hogares clasificados inicialmente como beneficiarios de la Asignación Universal por Hijo. Si bien esto podría significar que algunos hogares quedan excluidos del análisis a pesar de haber declarado correctamente más de un beneficiario por hogar, la comparación entre el verdadero número de hijos residentes y el número que se deriva del monto total del beneficio por hogar sugiere que el error de incluir estos casos sería más significativo que el error de excluirlos.
}

Asimismo, se siguió reduciendo el grupo de hogares clasificados como beneficiarios al excluir a los que no tenían niños. Esto respondió a la necesidad de disminuir la heterogeneidad de este grupo en relación con el grupo de control, que estaba formado por hogares elegibles para la asignación (los que, por ende, tienen niños).

Los hogares elegibles (grupo de control) son los que cumplen con todos los requisitos para recibir la Asignación Universal por Hijo, pero no la perciben. Como se mencionó, los beneficiarios potenciales son:

i) los hogares con hijos menores de 18 años cuyos jefes o cónyuges son asalariados no registrados (trabajadores informales) o trabajadores domésticos con ingresos inferiores al salario mínimo;

ii) los monotributistas sociales;

iii) los desempleados que no perciben seguro por desempleo, $\mathrm{y}$

iv) los trabajadores económicamente inactivos sin pensiones.

Por lo tanto, el análisis se limitará a los hogares (y sus miembros) con hijos que eran elegibles para la Asignación Universal por Hijo en 2009, diferenciándolos según hayan accedido al beneficio en 2010 (grupo de tratamiento) o no (grupo de control). Así, la condición

GRÁFICO 1

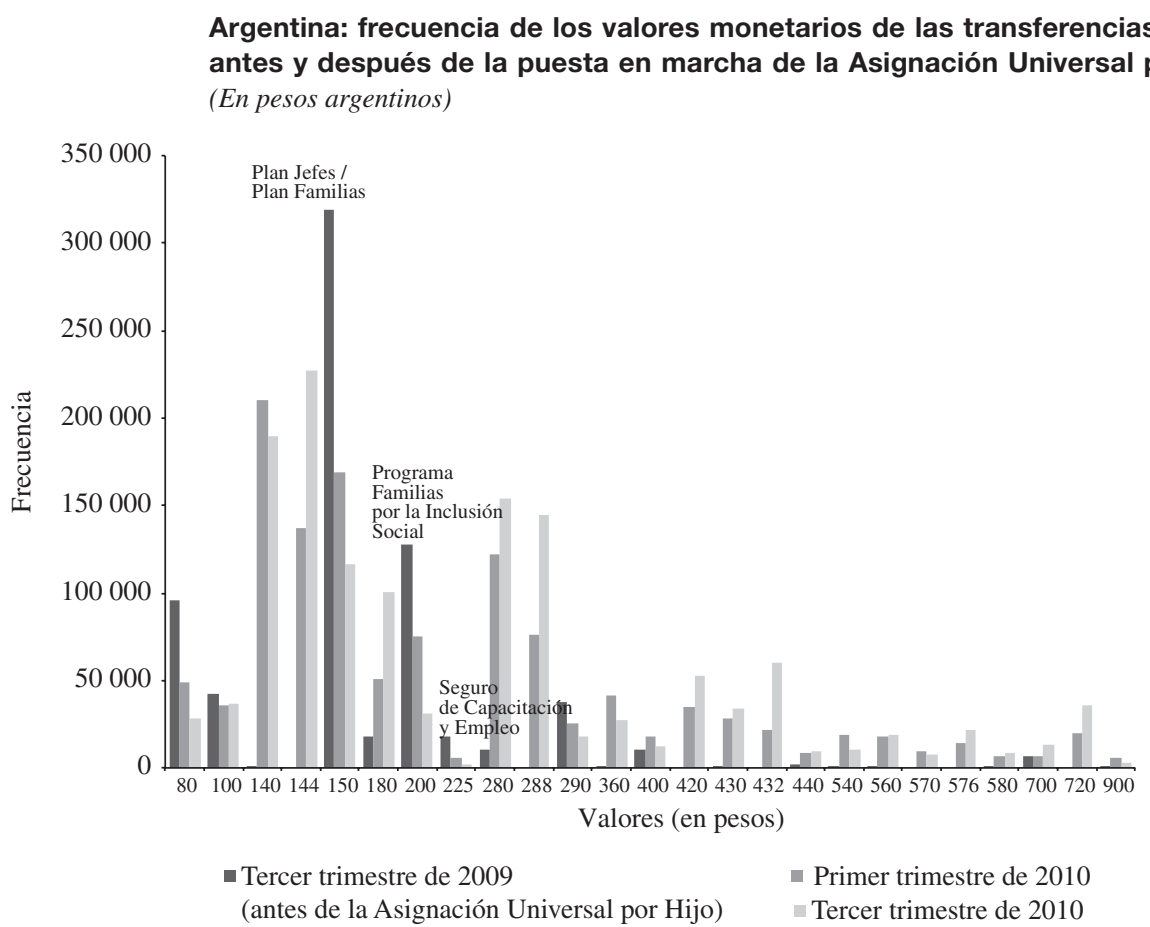

Fuente: elaboración propia sobre la base de datos de la Encuesta Permanente de Hogares (EPH) del Instituto Nacional de Estadística y Censos (INDEC). 
de elegibilidad corresponde a 2009, pero la condición de recepción corresponde a 2010.

Los hogares con información incompleta o con valores imputados a los ingresos individuales o familiares se excluyeron de la muestra. Además, los valores extremos de los ingresos familiares totales y sus componentes (los ingresos laborales y los no laborales) se suprimieron del análisis usando un método de estandarización robusto de los datos.

Dado que el objetivo es evaluar los efectos de la Asignación Universal por Hijo en el comportamiento laboral de los adultos, la muestra de análisis de los individuos está compuesta por grupos de personas en edad económicamente activa: hombres de 18 a 64 años y mujeres de 18 a 59 años. En ambos casos, la edad límite superior corresponde a la edad legal de jubilación.

Antes de finalizar esta sección, se deben aclarar algunos puntos referidos a la composición del grupo de hogares elegibles. Puesto que la Asignación Universal por Hijo se orienta a alcanzar una cobertura universal de los hogares con niños no cubiertos por el sistema de contribuciones, llama la atención que un grupo de hogares haya quedado fuera del programa durante el primer año, a pesar de reunir los requisitos. Si bien no se conocen por completo las causas de este fenómeno, hay ciertos factores que tal vez hayan influido.

Primero, como se señaló anteriormente, a fin de ingresar al programa tanto los niños como los padres deben tener documentos nacionales de identidad como prueba de identificación. Según algunos estudios cualitativos, al parecer este requisito representó una importante barrera de acceso, al menos en las primeras etapas del programa. En el caso de los hogares que tuvieron a su primer hijo, la demora en la afiliación del recién nacido pudo haber sido una razón adicional para que quedaran fuera del programa. Asimismo, otros problemas familiares pueden haber contribuido a esta situación (Pautassi, Arcidiácono y Straschnoy, 2013).

Además, algunas personas que al parecer estarían en condiciones de reunir los requisitos para obtener el beneficio según la EPH, podrían en realidad estar registradas como monotributistas $\mathrm{y}$, por consiguiente, no ser elegibles. Sin embargo, dado que es imposible identificar a este grupo en la encuesta, durante el análisis dichas personas permanecieron en el grupo elegible.

Según se analizó, la repercusión del programa puede variar de acuerdo con las limitaciones y preferencias de los hogares (Moffit, 2002; Skoufias y Parker, 2001). En particular, los hogares elegibles podrían quedar excluidos del programa debido a ciertos procesos administrativos o requisitos vinculados a las condiciones de acceso.
En tal sentido, es importante mencionar que no se utiliza el cumplimiento de las condicionalidades para determinar la elegibilidad de los hogares, por dos razones. En primer lugar, la EPH no brinda información completa sobre esta materia, particularmente en lo que respecta a los controles de salud. En cuanto a la educación, si bien en la encuesta se identifica la concurrencia de un niño a la escuela, si el niño no asiste, el hogar no necesariamente se vuelve no elegible porque aún puede recibir la asignación por otro menor que sí concurre. No obstante, no es posible identificar en la encuesta al menor por el cual el hogar recibe el beneficio. De todas maneras, el 90\% de los hogares elegibles seguirían siéndolo si se impusiera la restricción de que cada hijo que vive en el hogar debe asistir al colegio. En segundo lugar, el control de las condiciones fue más relajado en las primeras etapas del programa y solo se hizo más riguroso posteriormente.

Como se hizo presente, la evaluación se refiere al primer año de implementación del programa y, por ende, la existencia de un grupo de hogares elegibles no beneficiarios también podría deberse a demoras en el registro. La distancia entre los hogares y las oficinas administrativas del programa pudo haber sido otro factor disuasivo para la afiliación en las primeras etapas, antes de la puesta en marcha de medidas destinadas a alcanzar a la población de las zonas más alejadas.

Cabe agregar dos temas adicionales. Primero, para identificar con precisión el impacto del programa se requiere de la ausencia de efectos de anticipación (Ashenfelter's Dip), es decir, el grupo elegible no debe cambiar su comportamiento porque anticipa la ejecución del programa9. Dado que el anuncio de la Asignación Universal por Hijo fue totalmente inesperado, que se puso en marcha con mucha rapidez, que a noviembre de 2009 —el primer mes de operación- el programa ya había cubierto a 3,3 millones de niños y que este número se ha mantenido relativamente estable (en 3,5 millones), puede asumirse que no hubo en la población efectos significativos de anticipación destinados a ganar elegibilidad y acceso al programa, lo que podría haber ocasionado un sesgo de selección en esta evaluación.

Segundo, lamentablemente, dada la estructura de panel de corto plazo de la EPH, no es posible controlar si los hogares de los grupos de tratamiento y de control muestran tendencias similares en las variables de resultado antes de la puesta en marcha del programa, como sugiere, por ejemplo, Duflo (2001).

${ }^{9}$ Ashenfelter (1978) y Heckman y Smith (1999). 


\section{VI}

\section{Estadísticas descriptivas}

En esta sección se presentan las características de los beneficiaros de la Asignación Universal por Hijo (grupo de tratamiento), que luego se comparan con los hogares elegibles no beneficiarios (grupo de control) antes de la implementación del programa.

\section{Características de los beneficiarios}

En el cuadro A.1 del anexo se resumen las características demográficas y laborales de los beneficiarios de la Asignación Universal por Hijo y sus familias en $2010^{10}$. Con fines comparativos, en el cuadro se incluyen además a las personas en edad económicamente activa que viven en hogares no beneficiarios. Aproximadamente el 58\% de los beneficiarios son cónyuges y el $34 \%$ son jefes de hogar. Como es de esperar, las cifras varían significativamente por género, ya que casi un $90 \%$ de los hombres son jefes de hogar, mientras que un $64 \%$ de las mujeres son cónyuges. Las mujeres conforman la gran mayoría de los beneficiarios (89\%), lo que podría deberse, al menos en parte, a la reasignación de beneficiarios de programas anteriores de transferencias públicas, entre los que había un elevado número de mujeres. Además, como ya se dijo, las disposiciones del programa dan prioridad a las madres para recibir el beneficio. El relativamente alto nivel de informalidad entre las mujeres, en promedio, también podría ser un factor vinculado a este resultado.

El promedio de edad de los beneficiarios es de 35 años, siendo las mujeres más jóvenes que los hombres. Los niveles educativos son bastante bajos en los dos casos: rondan los 9,4 años de escolaridad en el caso de los hombres y los 9,6 en el caso de las mujeres. En cuanto a la población general no beneficiaria en edades económicamente activas, las cifras son de 10,9 y 11,8 años de escolaridad para hombres y mujeres, respectivamente.

\footnotetext{
${ }^{10} \mathrm{Si}$ bien los datos de la EPH, que abarca a todo el país, muestran un número menor de beneficiarios respecto del total que presentan los registros administrativos, la composición de la población en términos de variables personales es muy similar en ambas fuentes de información. La subestimación de beneficiarios se debe —al menos en parte- a que la EPH no está específicamente diseñada para identificar este tipo de transferencias. Al mismo tiempo, extender la encuesta a todo el país podría de por sí provocar algunos errores. No obstante, algunos autores como Galasso y Ravallion (2004) emplearon la misma fuente de información para evaluar el PJJHD, también en un contexto de subestimación del número de beneficiarios.
}

Con respecto a la situación de empleo de los beneficiarios, la proporción de trabajadores empleados y de inactivos resultó similar. Sin embargo, entre las mujeres el $52 \%$ eran inactivas y el $42 \%$ estaban empleadas, mientras que en el caso de los hombres estas cifras alcanzaban al $5 \%$ y el $90 \%$, respectivamente. En promedio, las mujeres beneficiarias trabajan 27 horas semanales y los hombres 43 horas. La participación laboral es, por consiguiente, menor en el caso femenino, tanto en términos de la tasa de actividad como de la carga horaria.

En promedio, los hogares beneficiarios tienen 4,7 miembros y los no beneficiarios, 4,4 ; en cuanto a los niños, el número es de 2,5 y de 2,0, respectivamente. A pesar del beneficio, la incidencia de la pobreza en esos hogares era todavía muy elevada en 2010: aproximadamente el $64 \%$ de los hogares beneficiarios eran pobres y un $18 \%$ eran extremadamente pobres ${ }^{11}$. En el caso de los hogares no beneficiarios, estas cifras eran del $18 \%$ y el $7 \%$, respectivamente.

Finalmente, en 2010, la Asignación Universal por Hijo cubrió aproximadamente a dos niños por hogar beneficiario, lo que representa unos 300 pesos argentinos (75 dólares). Este valor corresponde a alrededor del $40 \%$ del total de los ingresos familiares netos de transferencias. $\mathrm{Si}$ bien este beneficio es considerable en relación con los ingresos propios de estos hogares, la brecha de pobreza seguía siendo muy amplia tras la recepción del beneficio (en torno del 40\%).

\section{Comparación entre los hogares beneficiarios y los hogares no beneficiarios elegibles en 2009 (línea de base)}

Como puede observarse en el cuadro A.2 del anexo, los hogares que recibieron el beneficio en 2010 tienen familias más grandes y con mayor número de niños que los no beneficiarios observados en 2009. Además, los ingresos familiares son significativamente más bajos. Las

\footnotetext{
11 Los hogares se identifican como pobres si sus ingresos totales se ubican por debajo de la línea de pobreza. La línea de pobreza de 2009 y 2010 se construyó mediante una actualización del valor registrado en 2007, empleando la variación del índice oficial de precios al consumidor calculado en nueve provincias del país. Esta decisión se basó en la controversia surgida acerca del índice de precios al consumidor (IPC) publicado por el INDEC.
} 
funciones de densidad de kernel de los ingresos familiares per cápita muestran claramente que la distribución de los hogares elegibles que accedieron a la Asignación Universal por Hijo está desplazada hacia la izquierda en relación con el resto de los hogares, seguidos por los hogares elegibles no beneficiarios y luego por los hogares no beneficiarios y no elegibles con niños y los hogares solo con miembros adultos (véase el gráfico 2). Esto sugiere que la asignación estuvo dirigida, principalmente, a los hogares que se encuentran en el extremo inferior de la distribución de ingresos.

Debido a sus menores niveles de ingresos familiares, los hogares beneficiarios mostraban tasas de pobreza y extrema pobreza más elevadas. En 2009, el 74\% de los hogares eran pobres, mientras que en los hogares no beneficiarios la cifra alcanzaba al $65 \%$. En el caso de la extrema pobreza, las cifras de los hogares beneficiarios y de los no beneficiarios eran del $31 \%$ y el $26 \%$, respectivamente.

Dado que alrededor de un $92 \%$ de los beneficiarios son jefes de hogar o cónyuges, en el cuadro A. 2 del anexo se comparan las características de estos individuos con las de sus homólogos en los hogares no beneficiarios. No se observaron diferencias estadísticamente significativas en el porcentaje de cónyuges mujeres entre los grupos.
Tampoco fueron significativas las diferencias de los porcentajes de jefatura de hogar femenina entre el grupo de tratamiento y el grupo de control.

En los hogares que accedieron al beneficio en 2010, tanto los jefes de hogar como los cónyuges tenían, en promedio, menos edad en comparación con el grupo de hogares de 2009. El nivel educativo medio de los jefes y cónyuges también era significativamente más bajo en los hogares beneficiarios que en el otro grupo.

Finalmente, no se encontraron diferencias significativas entre los cónyuges de ambos grupos en lo que respecta a la participación y la composición laboral. El comportamiento de los jefes de ambos tipos de hogares con relación a estas variables tampoco fue diferente. Sin embargo, se observa lo contrario en cuanto a la carga horaria de los cónyuges: en 2009, los que vivían en hogares beneficiarios trabajaban, en promedio, 5,8 horas menos por semana que los de los hogares no beneficiarios.

En resumen, los dos grupos mostraron diferencias en algunas variables observables antes de que se lanzara el programa. Estas disparidades se tomarán en cuenta en el análisis econométrico, con el fin de estimar con precisión los efectos de la Asignación Universal por Hijo en el funcionamiento del mercado laboral.

GRÁFICO 2

Argentina: curvas de densidad de kernel de los ingresos familiares per cápita, tercer trimestre de 2009

(En pesos argentinos)

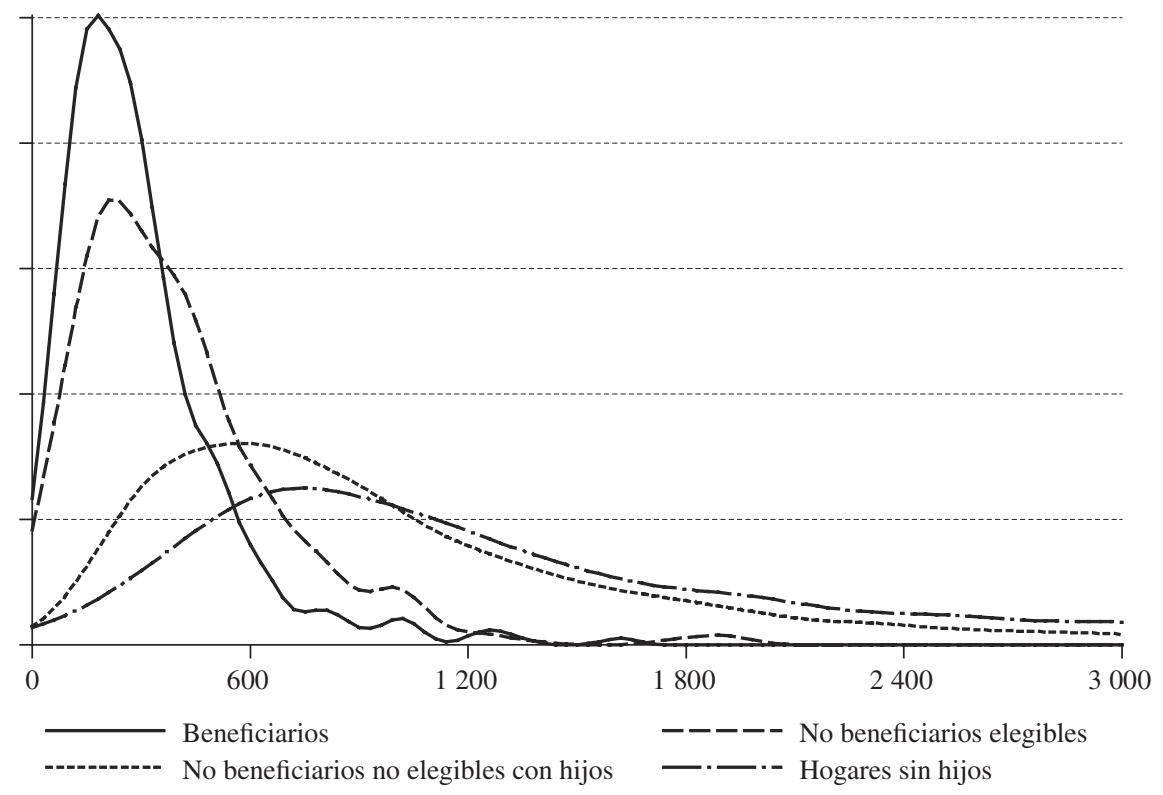

Fuente: elaboración propia sobre la base de datos de la Encuesta Permanente de Hogares (EPH) del Instituto Nacional de Estadística y Censos (INDEC). 


\section{VII}

\section{Resultados econométricos}

En esta sección se analizan los resultados econométricos derivados del estimador de diferencias en diferencias (DD), calculado a partir de la base de microdatos conteniendo los paneles correspondientes a los tres primeros trimestres de 2009 y 2010. Como se apuntó previamente, este estimador se aplica a todos los hogares con hijos que eran elegibles en 2009, de los cuales algunos recibieron el beneficio en 2010 y otros no accedieron al programa.

El análisis se efectúa en tres niveles de comparación: i) los hogares beneficiarios en comparación con los hogares no beneficiarios elegibles; ii) los miembros de cada uno de los hogares (beneficiarios y no beneficiarios), diferenciados por género y por su condición de jefe o cónyuge, y iii) los beneficiarios directos en relación con individuos comparables que viven en hogares del grupo de control ${ }^{12}$.

\section{Hogares beneficiarios en comparación con hogares elegibles no beneficiarios}

En el cuadro A. 3 del anexo se presentan los resultados de las estimaciones con respecto a los hogares. En cada variable de resultado se muestra el cambio medio del grupo de tratamiento y el grupo de control, el efecto medio del tratamiento en los tratados, los errores estándar ${ }^{13}$, los valores $p$ y el número de observaciones incluidas en cada grupo.

Si bien el signo de los parámetros del efecto medio del tratamiento en los tratados correspondientes a las variables del mercado laboral sugiere que la Asignación Universal por Hijo tiene un efecto negativo en las decisiones sobre participación económica -medidas por la proporción de miembros activos respecto del total de adultos del hogar- y en la tasa de empleo de los hogares, estos cambios no son estadísticamente significativos en ninguna de las dos alternativas de emparejamiento utilizadas: el método de vecino más cercano y la regresión lineal local. Asimismo, no se observa un efecto significativo en la incidencia del

\footnotetext{
12 No se incluyen los resultados de las estimaciones de los modelos logit utilizadas para obtener los índices de propensión, debido a limitaciones de espacio. Sin embargo, se encuentran disponibles previa solicitud.

13 Los errores estándares teóricos también se calcularon, pero no se presentan aquí porque no hubo diferencias significativas con respecto a los errores estándar obtenidos mediante la técnica de bootstrap.
}

desempleo ni en el promedio de horas trabajadas por los miembros del hogar empleados.

De la misma manera, las diferencias entre los hogares beneficiarios y los del grupo de control en cuanto a la evolución de los ingresos familiares totales y per cápita no son estadísticamente diferentes de cero. No obstante, este patrón común observado en la dinámica del total de ingresos de ambos grupos de hogares es, de hecho, el resultado de mayores aumentos de los ingresos no laborales, que superan el relativamente débil dinamismo de los ingresos laborales en los hogares beneficiarios en comparación con el grupo de control. El coeficiente negativo asociado con la estimación del efecto medio de la Asignación Universal por Hijo, en los ingresos laborales de sus beneficiarios, resulta estadísticamente relevante considerando un nivel de significancia del 10\%, y empleando la técnica de emparejamiento asociada con regresiones lineales locales. Sin embargo, al considerar los ingresos no laborales, los efectos resultan positivos y estadísticamente significativos al $1 \%$ en las dos alternativas de emparejamiento contempladas.

En resumen, la falta de significatividad estadística de los efectos estimados en variables asociadas con el mercado laboral parece sugerir que la implementación del programa no ha creado ningún desincentivo importante entre los adultos en el corto plazo (considerando que los datos de panel utilizados en este estudio solo incluyen el seguimiento a los hogares durante unos pocos trimestres). Sin embargo, a nivel de los hogares, esta situación podría ser el resultado neto de los distintos efectos de la asignación en sus miembros. A fin de analizar estos hallazgos con mayor detalle, a continuación se presentan los resultados de las estimaciones efectuadas separadamente para los distintos miembros adultos de los hogares.

\section{Comparación entre los miembros de hogares beneficiarios y los miembros de hogares no beneficiarios elegibles}

En el cuadro 2 se presenta la composición de los hogares beneficiarios por género y la posición de los miembros adultos dentro del hogar. Como puede observarse, los grupos más importantes son: i) el total de jefes y cónyuges; ii) el total de las mujeres; iii) el subgrupo de las mujeres cónyuges; iv) el total de los jefes; v) el subgrupo de las mujeres jefas, y vi) el subgrupo de los 
CUADRO 2

Argentina: composición de los miembros de hogares beneficiarios, por género y posición en el hogar, 2010

(En porcentajes)

\begin{tabular}{lccc}
\hline & Hombres & Mujeres & Total \\
\hline Jefes & 31 & 14 & 45 \\
Cónyuges & 3 & 30 & 33 \\
$\quad$ Subtotal & 34 & 44 & 78 \\
Niños & 8 & 10 & 18 \\
Otros miembros & 2 & 2 & 4 \\
$\quad$ Total & 44 & 56 & 100 \\
\hline
\end{tabular}

Fuente: elaboración propia sobre la base de datos de la Encuesta Permanente de Hogares (EPH) del Instituto Nacional de Estadística y Censos (INDEC).

hombres jefes. Por esta razón, en el nivel individual, el análisis se limitará a esos grupos, los que se compararán con sus contrapartes de los hogares del grupo de control.

En el cuadro A.4 del anexo se presentan las estimaciones econométricas correspondientes a estos grupos. En este nivel de análisis, los resultados son coherentes con los obtenidos en el nivel de los hogares, en cuanto a que la mayoría de los miembros de los hogares beneficiarios no se comporta de manera significativamente diferente de los correspondientes a los hogares del grupo de control. En particular, los efectos medios del tratamiento en los tratados relativos a la actividad y las condiciones de empleo resultaron no ser estadísticamente significativos para los miembros de todos los hogares y con las dos técnicas de emparejamiento utilizadas. No obstante, el aumento relativo observado en la tasa de desempleo de las cónyuges en los hogares beneficiarios es estadísticamente significativo al 5\% o al 10\%, dependiendo de la técnica de emparejamiento que se aplique.

Con relación al promedio de horas trabajadas (calculado solo sobre los individuos que en las dos observaciones estaban empleados), es notorio que si bien en los hogares beneficiarios el número de horas que trabajan las mujeres disminuye mientras que en los no beneficiarios ocurre lo contrario, las diferencias medias entre los dos grupos no son estadísticamente significativas, ni lo son en el caso del resto de los miembros considerados.

Respecto de las variaciones del ingreso familiar y sus fuentes, el mayor incremento de los ingresos no laborales registrado entre los hogares beneficiarios es una consecuencia de lo que ocurría con las mujeres en general y con las cónyuges en particular. Esto coincide con el hecho de que las mujeres representan alrededor de un $90 \%$ del total de beneficiarios de la asignación. La significatividad muy baja o nula que se observó en las diferencias de los ingresos laborales en el nivel de los hogares también se confirma en el nivel individual. Como resultado, las diferencias de los ingresos no laborales se trasladan a la dinámica de las brechas de los ingresos totales. De hecho, las mujeres en general y las cónyuges en particular (al aplicar el método de vecino más cercano) experimentan aumentos significativos de los ingresos individuales totales como consecuencia de recibir la asignación.

En síntesis, al igual que en los resultados anteriores, los hallazgos relativos a los miembros de los hogares no permiten concluir que recibir una transferencia monetaria como la Asignación Universal por Hijo representa un desincentivo en el corto plazo para participar en el mercado laboral o reduzca el número de horas trabajadas en el caso de las personas que siguen empleadas.

\section{Comparación entre las mujeres receptoras de la Asignación Universal por Hijo (jefas de hogar o cónyuges) y las mujeres de hogares elegibles no beneficiarios}

Por último, se evaluará la Asignación Universal por Hijo comparando el comportamiento de los beneficiarios con el de los miembros de los hogares del grupo de control. La diferencia respecto del ejercicio anterior es que allí se compara a los miembros de los hogares beneficiarios con los del grupo de control sin identificar a los beneficiarios del primer grupo.

Dado que casi todos los beneficiarios son mujeres, el análisis se restringirá a este subgrupo de personas. En particular, se evalúan las decisiones laborales y la generación de ingresos de todas las beneficiarias (y se comparan con las de las mujeres adultas de los hogares elegibles no beneficiarios) y luego se consideran las de las jefas de hogar y las cónyuges por separado. En los últimos dos casos, la comparación se realiza con respecto a las jefas de hogar y mujeres cónyuges de los hogares del grupo de control, respectivamente.

Los resultados, que se presentan en el cuadro A.5 del anexo, confirman nuevamente que la Asignación Universal por Hijo no tuvo efectos significativos en las decisiones laborales entre 2009 y 2010. En particular, este programa no parece haber alentado salidas netas hacia la inactividad, ni haber causado una reducción del número de horas trabajadas por las mujeres, y en consecuencia, no originó un descenso de su oferta de mano de obra (ni en las jefas de hogar ni en las cónyuges). Es importante destacar que el incremento relativo observado en la tasa de desempleo femenino en los hogares beneficiarios 
(aunque la diferencia entre los grupos fue significativa solo en un 5\% y un 10\%) resulta insignificante cuando el análisis se restringe a las beneficiarias mujeres. El efecto estimado es significativo solo en un nivel del $10 \%$ al aplicar la técnica de emparejamiento basada en regresiones lineales locales.

La ausencia de efectos significativos de la Asignación Universal por Hijo en las decisiones sobre el trabajo es coherente con el efecto nulo del programa en los ingresos laborales de las beneficiarias mujeres. Por el contrario, en el caso de los ingresos no laborales de las beneficiarias (en particular, de las que son cónyuges), se observa un estimado del efecto medio significativo

\section{VIII}

\section{Conclusiones}

La introducción de la Asignación Universal por Hijo representa un importante paso adelante hacia la superación de los retos que implica el cierre de la brecha existente en la cobertura de la protección social infantil en la Argentina. Este programa tiene una relación directa con el sistema de seguridad social contributiva, en el sentido de que extiende el sistema de asignaciones familiares para niños y adolescentes al que acceden los trabajadores de la economía formal.

Este es el primer estudio en que se miden los efectos de la asignación en la participación laboral, el empleo, el desempleo, las horas trabajadas y la generación de ingresos laborales y no laborales de los adultos, utilizando el estimador de diferencias en diferencias (DD) en conjunto con técnicas de emparejamiento basadas en la estimación de índices de propensión.

Teniendo en cuenta los resultados obtenidos, no es posible concluir que el programa haya generado desincentivos al trabajo entre los adultos miembros de los hogares beneficiados en los años 2009 y 2010 , en el sentido de alentarlos a dejar la fuerza laboral o a reducir el número de horas de trabajo. Tales resultados son coherentes con gran parte de la evidencia empírica sobre programas de transferencias similares de otros países latinoamericanos y altamente relevantes para la discusión acerca de la formulación de políticas públicas sociales en la región, teniendo en cuenta que se deben minimizar los potenciales efectos colaterales negativos de dichos programas en el mercado laboral y que las transferencias monetarias de este tipo han adquirido como resultado de la percepción del beneficio, lo que, en la ausencia de cambios negativos en otras fuentes de ingresos, produce aumentos netos del total de los ingresos recibidos por las beneficiarias ${ }^{14}$.

${ }^{14}$ Las estimaciones están basadas en la comparación de los grupos de hogares e individuos que permanecen en la muestra tras las exclusiones mencionadas en la sección III. Sin embargo, existen algunos beneficiarios en el soporte común cuyas probabilidades estimadas de ser tratados resultan próximas a cero. De acuerdo con la propuesta de Heckman, Ichimura y Todd (1997), se evaluaron diversas alternativas de corte sobre el soporte común para evitar el sesgo que podría surgir en las estimaciones al incluir estos casos. Los resultados apoyan las conclusiones previas. una relevancia creciente como parte constitutiva del sistema de protección social de América Latina. Sin embargo, conviene aclarar al menos cuatro puntos sobre estas conclusiones. Primero, a diferencia de otros estudios en que se realizan análisis de impactos como parte de una evaluación de este tipo de programas, no se realizó ninguna encuesta específicamente diseñada para este propósito en el país. Segundo, en la encuesta de hogares utilizada, los beneficiarios de la Asignación Universal por Hijo solo se identifican indirectamente. Tercero, podrían surgir diferencias en las características no observadas entre el grupo de tratamiento y el grupo de control, aunque la metodología empleada se orienta a reducir la probabilidad de este problema. Finalmente, en este artículo se cubre un período de tiempo relativamente corto y los efectos en la participación en el mercado laboral podrían cambiar si se analizara un horizonte temporal más prolongado.

Por lo tanto, es esencial contar con información fiable y actualizada para efectuar un seguimiento continuo y una evaluación precisa de los posibles efectos de la Asignación Universal por Hijo y otros programas de protección social. La Argentina presenta un rezago considerable en esta área, en comparación con otros países de la región que tienen niveles similares de ingresos y desarrollo de la seguridad social.

Asimismo, si bien la implementación de este programa marca una mejora significativa en la cobertura social de los niños, quedan importantes retos por resolver. Por ejemplo, el programa excluye a los trabajadores 
informales que ganan un monto superior al salario mínimo. En la medida en que la Asignación Universal por Hijo se considera una extensión del sistema de contribuciones, se debería progresar en la estandarización de los requisitos de los dos sistemas, sobre todo respecto del límite máximo de ingresos, que en la actualidad es considerablemente más alto en el sistema contributivo que en este programa.

Con respecto al cumplimiento de las condiciones para acceder al beneficio monetario, se debería tener en cuenta la disponibilidad de centros de salud y establecimientos educativos en los barrios donde residen los beneficiarios y las zonas cercanas, así como la calidad de los servicios que brindan. Por otra parte, se estableció que los beneficiarios de la Asignación Universal por Hijo no pueden participar en ningún otro programa social. Esta disposición parece razonable cuando el beneficio reemplaza a otros programas de transferencias monetarias que se orientan a necesidades similares, pero no toma en cuenta que estos tienen distintos objetivos: por ejemplo, algunos se destinan a mejorar la empleabilidad de los trabajadores desempleados o de los que se encuentran en la economía informal. En consecuencia, se debe avanzar hacia la integración y articulación de los varios componentes del sistema de protección social.

Por último, todas estas políticas deberían enmarcarse dentro de una estrategia económica de largo plazo construida sobre la base de una estructura de producción integrada que conduzca a una mayor eficiencia y competitividad sistémica y al incremento de la demanda laboral.

ANEXO

CUADRO A.1

Argentina: características de los beneficiarios y no beneficiarios de la Asignación Universal por Hijo, 2010

\begin{tabular}{|c|c|c|c|c|c|c|}
\hline \multirow{2}{*}{ Características } & \multicolumn{3}{|c|}{ Beneficiarios } & \multicolumn{3}{|c|}{ No beneficiarios (activos) } \\
\hline & Total & Mujeres & Hombres & Total & Mujeres & Hombres \\
\hline \multicolumn{7}{|l|}{ Relación familiar } \\
\hline Jefe & $33,6 \%$ & $26,8 \%$ & $88,5 \%$ & $41,9 \%$ & $19,8 \%$ & $69,3 \%$ \\
\hline Cónyuge o pareja & $57,9 \%$ & $64,3 \%$ & $6,2 \%$ & $34,9 \%$ & $57,8 \%$ & $6,7 \%$ \\
\hline Otros miembros & $8,5 \%$ & $8,9 \%$ & $5,3 \%$ & $23,2 \%$ & $22,4 \%$ & $24,1 \%$ \\
\hline Años de edad & 35,1 & 34,6 & 38,8 & 35,9 & 35,4 & 36,6 \\
\hline Años de educación & 9,6 & 9,6 & 9,4 & 11,4 & 11,8 & 10,9 \\
\hline \multicolumn{7}{|l|}{ Situación laboral } \\
\hline Empleados & $47,1 \%$ & $41,8 \%$ & $89,6 \%$ & $65,7 \%$ & $49,1 \%$ & $86,3 \%$ \\
\hline Desempleados & $6,6 \%$ & $6,7 \%$ & $5,6 \%$ & $5,3 \%$ & $5,5 \%$ & $5,2 \%$ \\
\hline Inactivos & $46,4 \%$ & $51,6 \%$ & $4,8 \%$ & $29,0 \%$ & $45,4 \%$ & $8,6 \%$ \\
\hline Horas trabajadas & 30,0 & 26,6 & 43,0 & 40,0 & 31,5 & 45,9 \\
\hline \multicolumn{7}{|l|}{ Género } \\
\hline Mujeres & $88,9 \%$ & & & $55,3 \%$ & & \\
\hline \multicolumn{7}{|l|}{ Número de miembros del hogar (en promedio) } \\
\hline Miembros de 0 a 5 años & 1,4 & & & 1,3 & & \\
\hline Miembros de 6 a 12 años & 1,6 & & & 1,4 & & \\
\hline Miembros de 13 a 17 años & 1,4 & & & 1,4 & & \\
\hline Miembros de 18 a 59 o 64 años & 2,2 & & & 2,3 & & \\
\hline Número de niños & 2,5 & & & 2,0 & & \\
\hline Total & 4,7 & & & 4,4 & & \\
\hline Hogares pobres & $63,7 \%$ & & & $26,1 \%$ & & \\
\hline Hogares en extrema pobreza & $17,9 \%$ & & & $7,1 \%$ & & \\
\hline Niños cubiertos por la Asignación Universal por Hijo & 2,1 & & & & & \\
\hline Monto de la asignación (en pesos argentinos) & 305,08 & & & & & \\
\hline Monto de la asignación en porcentajes del ingreso familiar & $38,5 \%$ & & & & & \\
\hline Brecha de pobreza (ingreso familiar neto) & $45,0 \%$ & & & & & \\
\hline Brecha de pobreza (ingreso familiar) & $38,0 \%$ & & & & & \\
\hline
\end{tabular}

Fuente: elaboración propia sobre la base de datos de la Encuesta Permanente de Hogares (EPH) del Instituto Nacional de Estadística y Censos (INDEC). 
CUADRO A.2

Argentina: características de los beneficiarios y no beneficiarios de la Asignación Universal por Hijo, 2009

\begin{tabular}{|c|c|c|c|c|}
\hline Características & No beneficiarios & Beneficiarios & \multicolumn{2}{|c|}{ Diferencia } \\
\hline \multicolumn{5}{|l|}{ Hogar } \\
\hline Miembros & 4,6 & 4,8 & $-0,13$ & \\
\hline Hijos & 2,3 & 2,6 & $-0,31$ & $* * *$ \\
\hline Ingreso total (en pesos argentinos) & 1253,5 & 1130,7 & 122,9 & $* * *$ \\
\hline Ingreso per cápita (en pesos argentinos) & 290,3 & 251,6 & 38,7 & $* * *$ \\
\hline Ingreso laboral (en pesos argentinos) & 1112,2 & 979,9 & 132,2 & $* * *$ \\
\hline Ingreso no laboral (en pesos argentinos) & 141,4 & 150,7 & $-9,4$ & \\
\hline Pobres & $65,1 \%$ & $74,4 \%$ & $-9,3$ p.p. & $* * *$ \\
\hline En extrema pobreza & $26,2 \%$ & $30,5 \%$ & $-4,4$ p.p. & $* *$ \\
\hline \multicolumn{5}{|l|}{ Jefes } \\
\hline Mujeres & $64,9 \%$ & $68,5 \%$ & $-3,5$ p.p. & \\
\hline Años de edad & 39,9 & 37,5 & 2,3 & $* * *$ \\
\hline Años de educación & 9,3 & 9,0 & 0,3 & ** \\
\hline \multicolumn{5}{|l|}{ Situación laboral } \\
\hline Empleados & $80,5 \%$ & $79,4 \%$ & 1,1 p.p. & \\
\hline Desempleados & $6,5 \%$ & $8,5 \%$ & $-2,0$ p.p. & * \\
\hline Inactivos & $13,0 \%$ & $12,1 \%$ & 0,9 p.p. & \\
\hline Horas trabajadas & 42,0 & 42,2 & $-0,2$ & \\
\hline \multicolumn{5}{|l|}{ Cónyuges } \\
\hline Mujeres & $8,2 \%$ & $7,8 \%$ & 0,4 p.p. & \\
\hline Años de edad & 36,1 & 34,1 & 1,9 & $* * *$ \\
\hline Años de educación & 10,0 & 9,4 & 0,6 & $* * *$ \\
\hline \multicolumn{5}{|l|}{ Situación laboral } \\
\hline Empleados & $41,1 \%$ & $41,2 \%$ & $-0,1$ p.p. & \\
\hline Desempleados & $6,1 \%$ & $5,1 \%$ & 1,0 p.p. & \\
\hline Inactivos & $52,8 \%$ & $53,7 \%$ & $-0,9$ p.p. & \\
\hline Horas trabajadas & 33,6 & 27,8 & 5,8 & $* * *$ \\
\hline
\end{tabular}

Fuente: elaboración propia sobre la base de datos de la Encuesta Permanente de Hogares (EPH) del Instituto Nacional de Estadística y Censos (INDEC).

Nota: $* * *=$ valor $\mathrm{p}<0,01 ; * *=$ valor $\mathrm{p}<0,05 ; *=$ valor $\mathrm{p}<0,1 ; \mathrm{p} . \mathrm{p} .=$ puntos porcentuales 


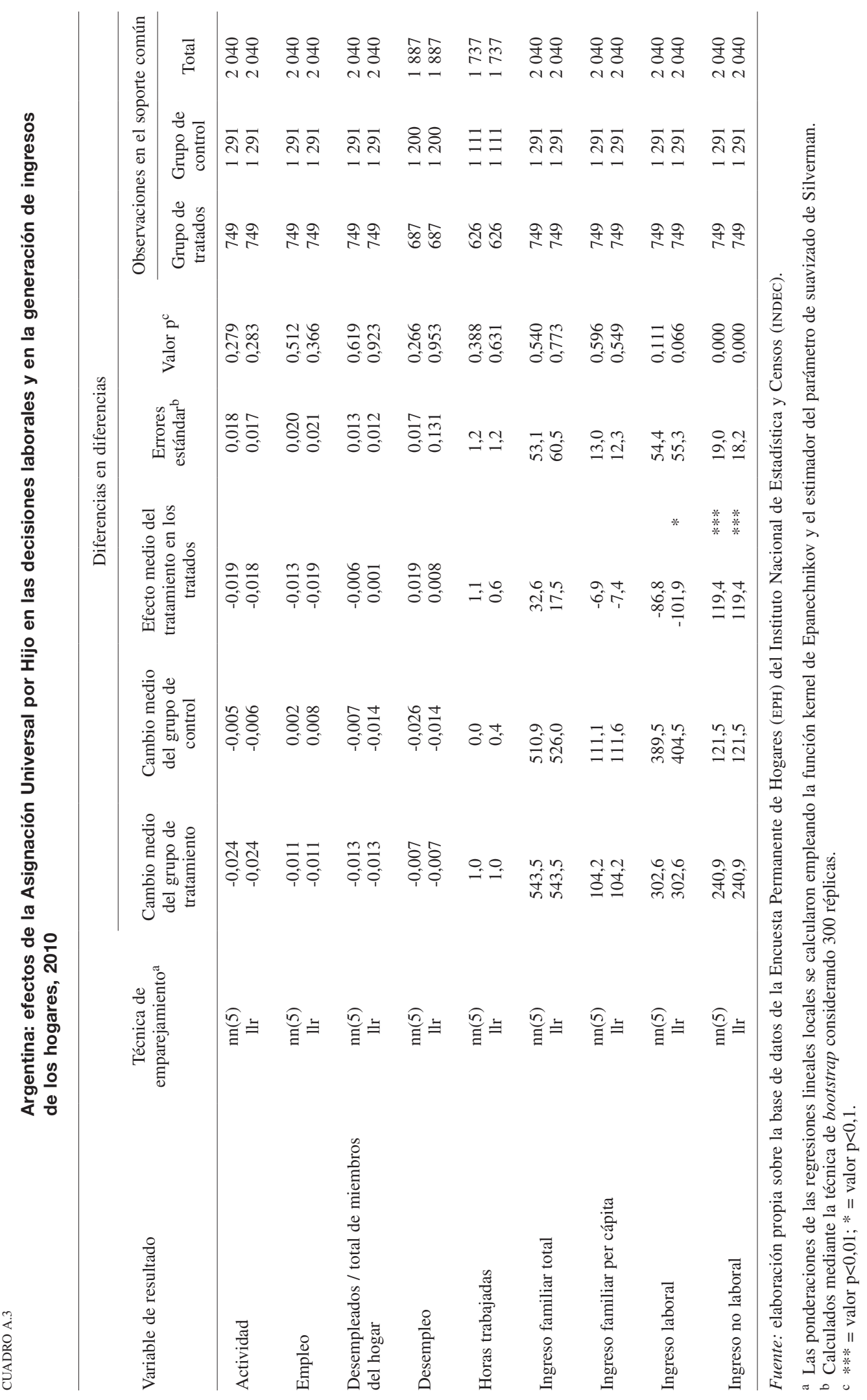




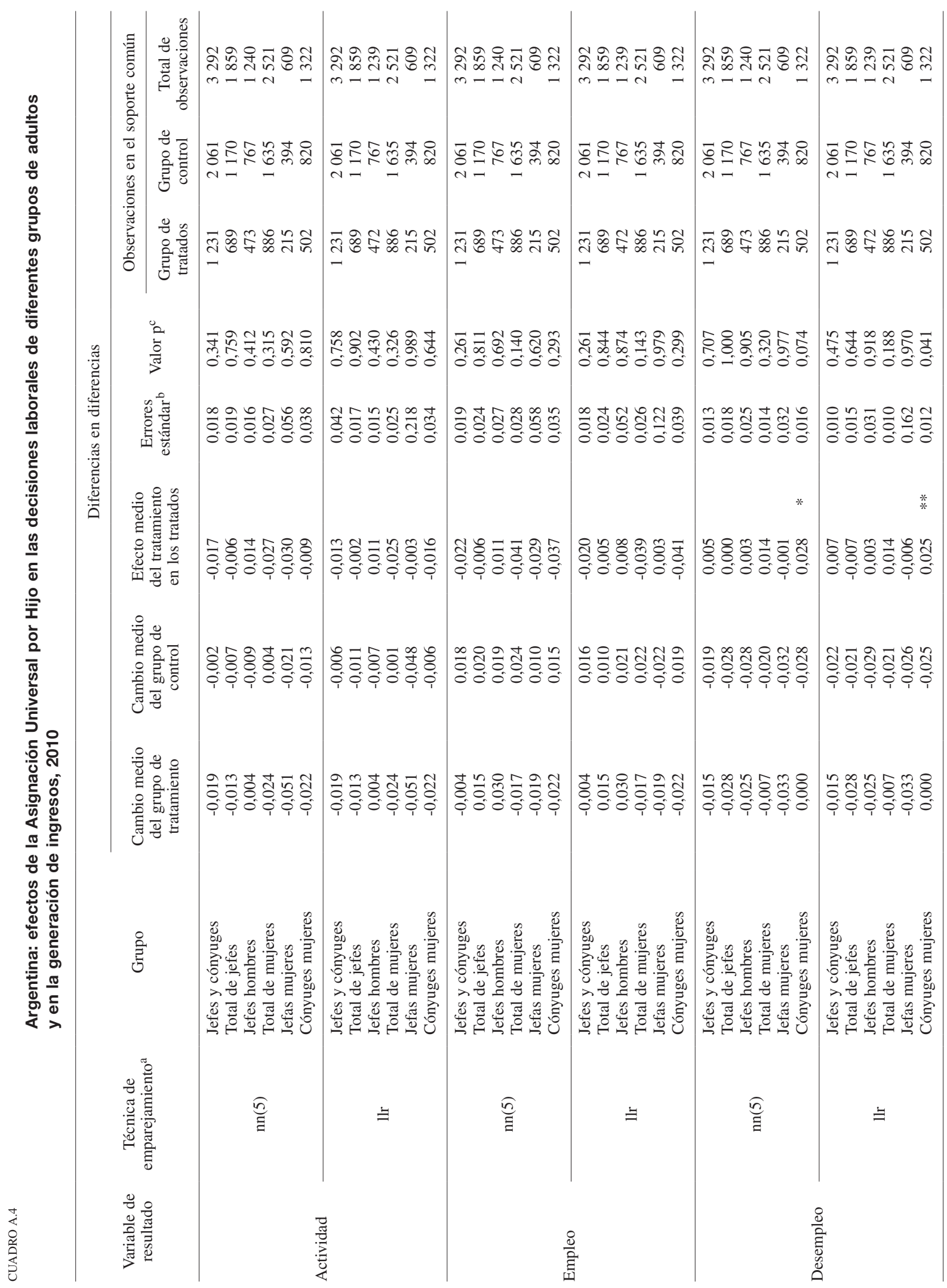




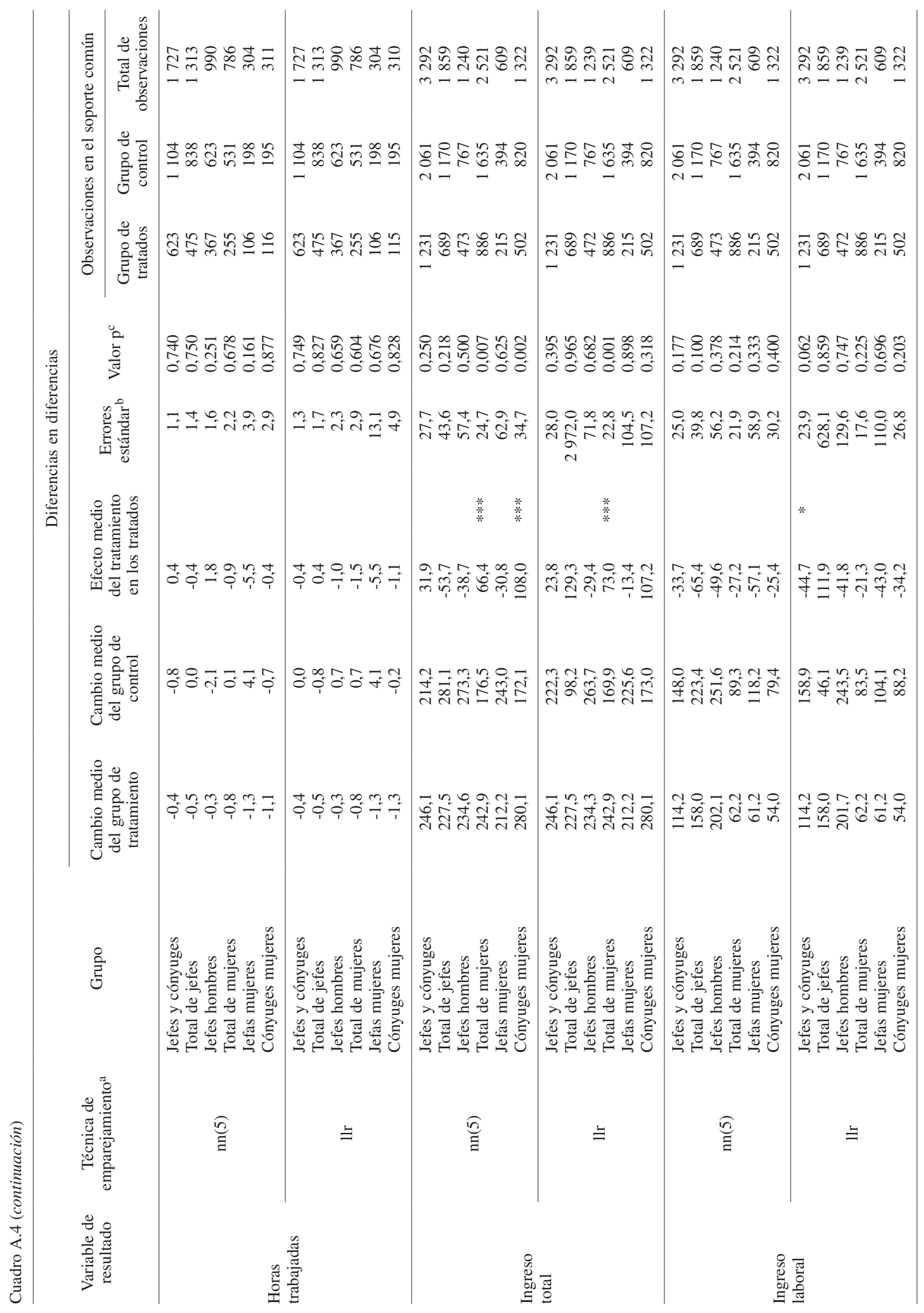




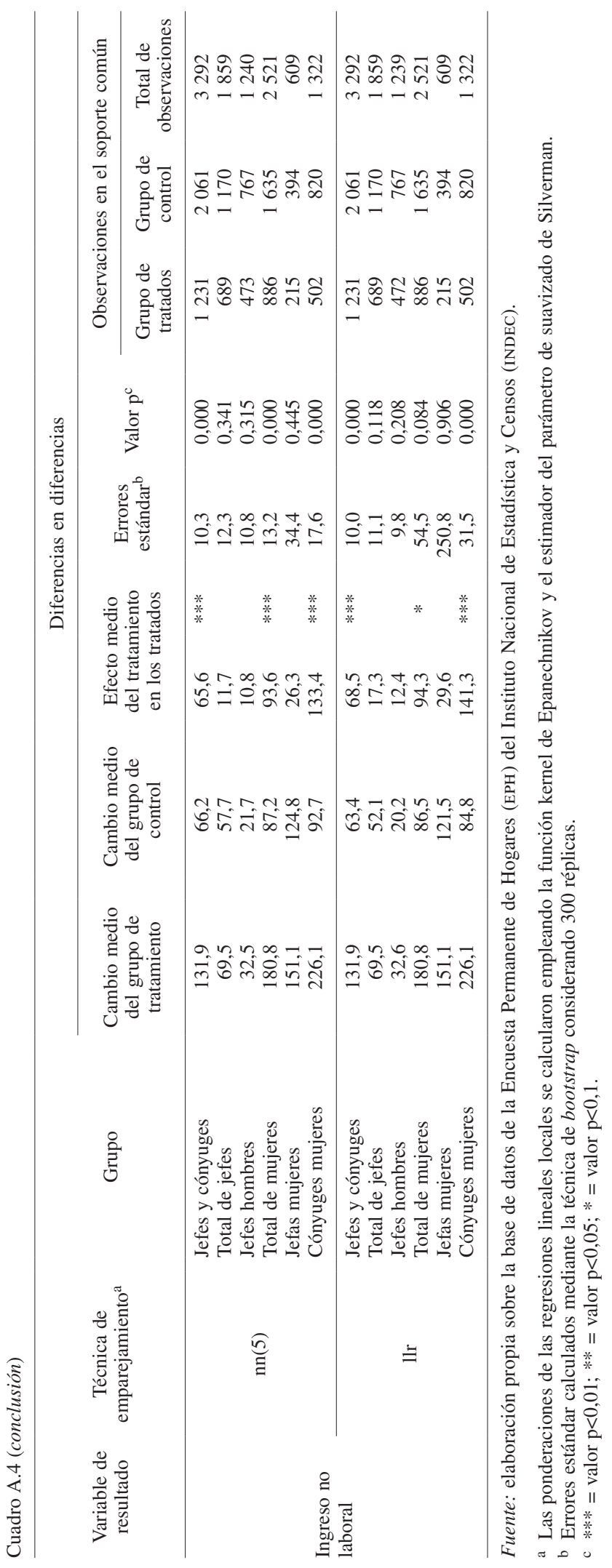




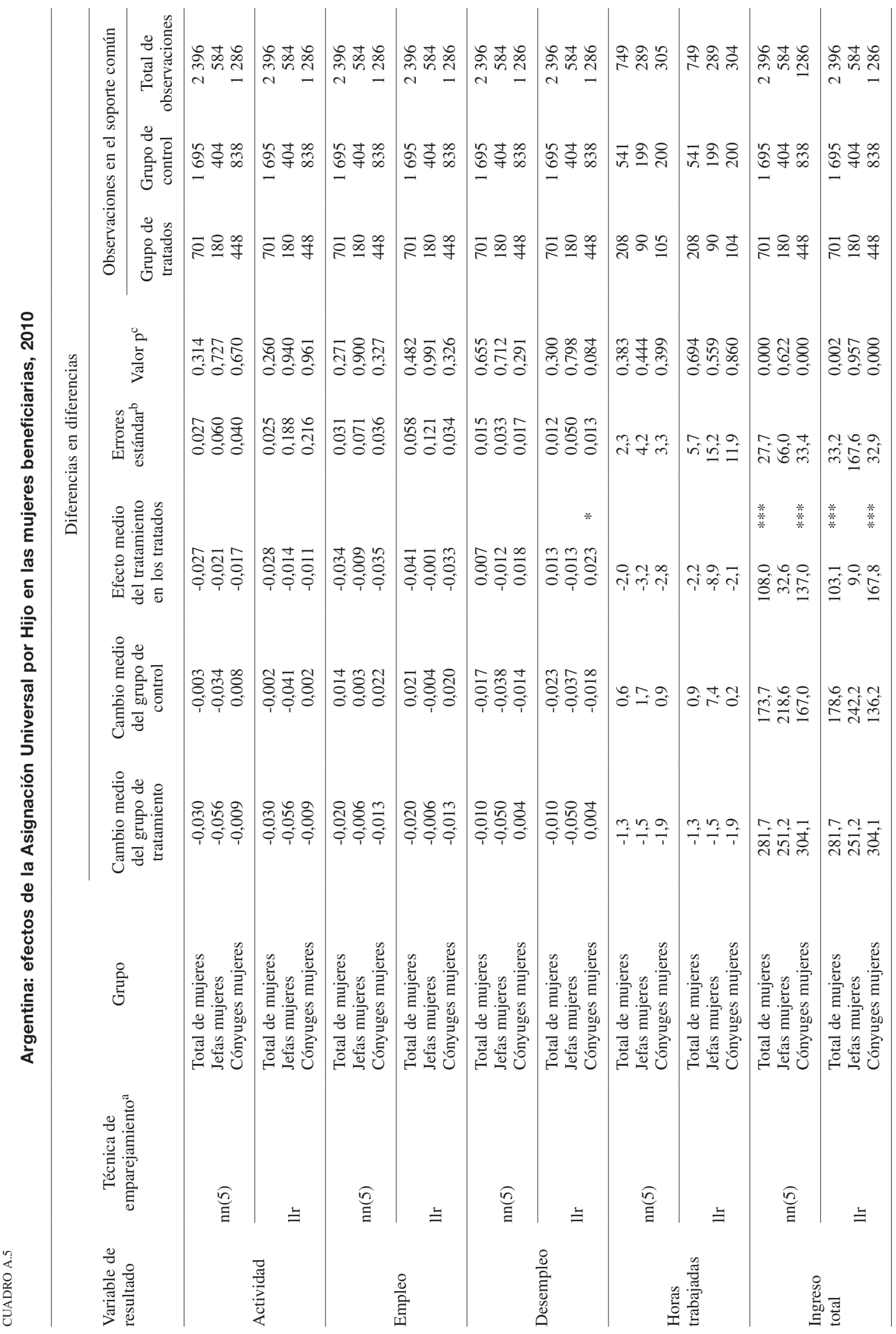




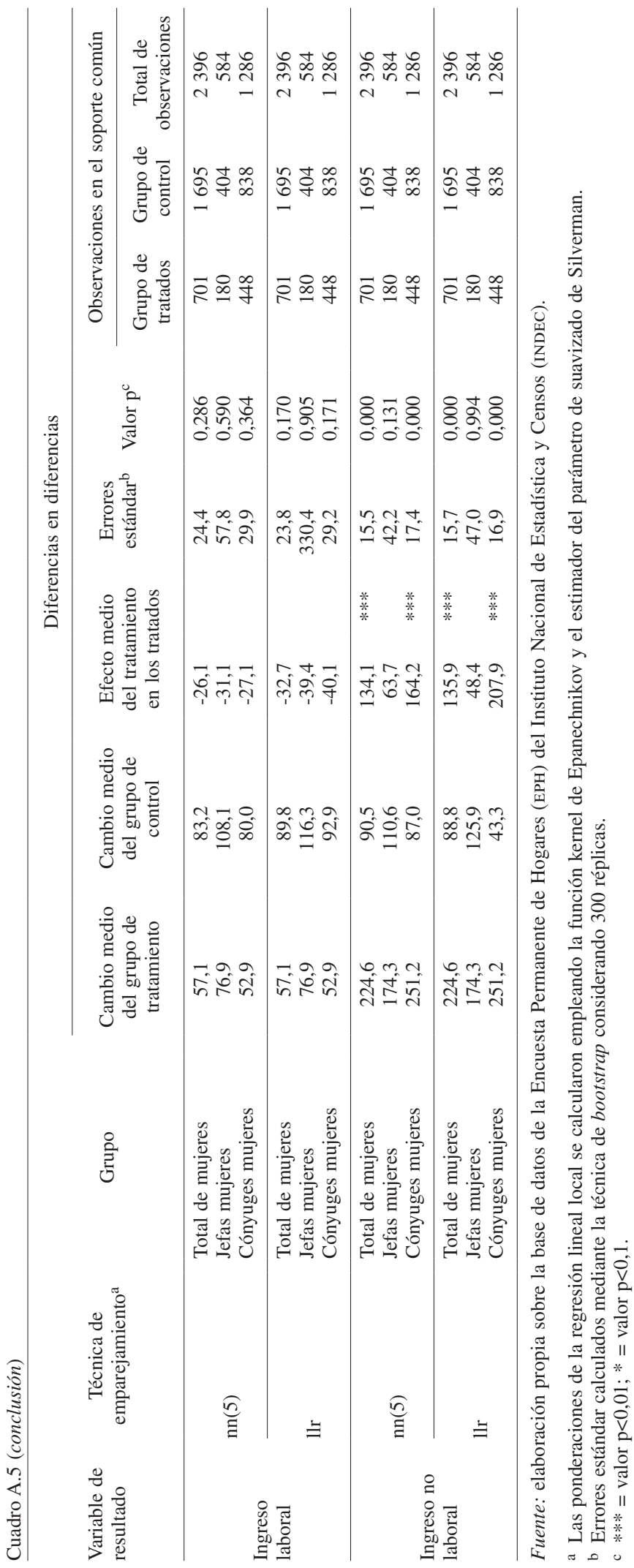




\section{Bibliografía}

Agis, E., C. Cañete y D. Panigo (2010), "El impacto de la asignación universal por hijo en Argentina", Centro de Estudios para el Desarrollo Argentino (CENDA)/Programa de Formación Popular en Economía (PROFPE)/Centro de Estudios e Investigaciones Laborales - Programa de Investigaciones Económicas sobre Tecnología, Trabajo y Empleo (CEIL-PIETTE).

Alzúa, M., G. Cruces y L. Ripani (2010), "Welfare programmes and labour supply in developing countries. Experimental evidence from Latin America", Documento de trabajo, $\mathrm{N}^{\circ}$ 95, La Plata, Centro de Estudios Distributivos, Laborales y Sociales (CEDLAS), Universidad Nacional de La Plata.

Amarante, V., M. Ferrando y A. Vigorito (2011), "School attendance, child labour and cash transfers: an impact evaluation of PANES", Working Paper, $\mathrm{N}^{\circ} 22 / 11$, Poverty and Economic Policy Network-PIERI.

Ashenfelter, O. (1978), "Estimating the effect of training programmes on earnings", Review of Economics and Statistics, vol. 60, $\mathrm{N}^{\circ} 1$, Cambridge, Massachusetts, MIT Press.

Bertranou, F. y R. Maurizio (2012), "Semi-conditional cash transfers in the form of family allowances for children and adolescents in the informal economy in Argentina", International Social Security Review, vol. 65, N ${ }^{\circ}$ 1, Ginebra, Asociación Internacional de la Seguridad Social.

Duflo, E. (2001), "Schooling and labour market consequences of school construction in Indonesia: evidence from an unusual policy experiment", American Economic Review, vol. 91, $\mathrm{N}^{\circ} 4$, Nashville, Tennessee, American Economic Association.

Fan, J. (1992), "Design-adaptive nonparametric regression", Journal of the American Statistical Association, vol. 87, $\mathrm{N}^{\circ} 420$, Alexandria, American Statistical Association, diciembre.

Ferro, A., A. Kassouf y D. Levison (2010), "The impact of conditional cash transfer programmes on household work decisions in Brazil", Child Labour and the Transition between School and Work, Research in Labour Economics, R. Akee, E. Edmonds y K. Tatsiramos (eds.), Emerald Group Publishing Limited.

Ferro, A. y A. Nicollela (2007), "The Impact of Conditional Cash Transfers Programmes on Household Work Decision in Brazil" [en línea] http://www.iza.org/conference_files/worldb2007/ ferro_a3468.pdf.

Foguel, M. y R. Paes de Barros (2010), "The effects of conditional cash transfer programmemes on adult labour supply: an empirical analysis using a time-series-cross-section sample of Brazilian municipalities", Estudos Econômicos, vol. 40, N 2 , São Paulo, Universidad de São Paulo.

Galasso, M. (2006), "With their effort and one opportunity: alleviating extreme poverty in Chile", Documento de Trabajo, Washington, D.C., Banco Mundial.

Galasso, M. y M. Ravallion (2004), "Social protection in a crisis: Argentina's Plan Jefes y Jefas", The World Bank Economic Review, vol. 18, $\mathrm{N}^{\circ}$ 3, Washington, D.C., Banco Mundial.

Gammage, S. (2010), "Time pressed and time poor: unpaid household work in Guatemala", Feminist Economics, vol. 16, $\mathrm{N}^{\circ} 3$, Taylor \& Francis.

Garganta, S. y L. Gasparini (2012), "El impacto de un programa social sobre la informalidad laboral: El caso de la AUH en Argentina", Documento de Trabajo, $\mathrm{N}^{\circ} 133$, La Plata, Centro de Estudios Distributivos, Laborales y Sociales (CEDLAS), Universidad Nacional de La Plata.

Gasparini, L. y G. Cruces (2010), "Las asignaciones universales por hijo: Impacto, discusión y alternativas", Documento de Trabajo, $N^{\circ}$ 102, La Plata, Centro de Estudios Distributivos, Laborales y Sociales (CEDLAS), Universidad Nacional de La Plata.

Heckman, J., H. Ichimura y P. Todd (1998) "Matching as an Econometric Evaluation Estimator", The Review of Economic Studies, vol. 65, $\mathrm{N}^{\circ}$ 2, Oxford, Oxford University Press.
(1997), "Matching as an econometric evaluation estimator: evidence from evaluating a job training programme", The Review of Economic Studies, vol. 64, N ${ }^{\circ}$, Oxford, Oxford University Press.

Heckman, J. y J. Smith (1999), "The pre-programme earnings dip and the determinants of participation in a social programme. Implications for simple programme evaluation strategies", The Economic Journal, vol. 109, ํㅜㄴ 457 , Wiley.

Killingsworth, M. (1983), Labour supply, Cambridge, Massachusetts, Cambridge University Press.

Medeiros, M., T. Britto y F. Veras Soares (2008), "Targeted cash transfer programmemes in Brazil: BPC and the Bolsa Familia", Working Paper, $\mathrm{N}^{\circ} 46$, Brasilia, Centro Internacional de Políticas para el Crecimiento Inclusivo.

Moffit, R. (2002), "Welfare programmes and labour supply", NBER Working Paper, $\mathrm{N}^{\circ}$ 9168, Cambridge, Massachusetts, National Bureau of Economic Research.

OIT (Organización Internacional del Trabajo) (2010), Aportes para la construcción de un piso de protección social en Argentina: El caso de las asignaciones familiares, Buenos Aires, Oficina de País de la oIT para la Argentina.

Parker, S. y E. Skoufias (2000), "The impact of Progresa on work, leisure, and time allocation", Documento de trabajo, Washington, D.C., Instituto Internacional de Investigación sobre Políticas Alimentarias.

Pautassi, L., P. Arcidiácono y M. Straschnoy (2013), "Asignación universal por hijo para la protección social de la Argentina. Entre la satisfacción de necesidades y el reconocimiento de derechos", Serie Políticas Sociales, N ${ }^{\circ} 184$ (LC/L.3662), Santiago de Chile, Comisión Económica para América Latina y el Caribe (CEPAL).

Ravallion, M. y Q. Wodon (2000), "Does child labour displace schooling? Evidence on behavioural responses to an enrollment subsidy", The Economic Journal, vol. 110, N 462, Royal Economic Society, marzo.

Roca, E. (2010), "Ingreso universal por hijo para protección social", documento presentado en el taller internacional "Compartiendo experiencias innovadoras sobre el piso de protección social", Turín, Centro Internacional de Formación.

Skoufias, E. y V. di Maro (2008), "Conditional cash transfers, adult work incentives, and poverty", Journal of Development Studies, vol. 44, $\mathrm{N}^{\circ}$ 7, Taylor \& Francis, julio.

Skoufias, E. y S. Parker (2001), "Conditional cash transfers and their impact on child work and schooling", FCND Discussion Paper, $\mathrm{N}^{\circ} 123$, Washington, D.C., Instituto Internacional de Investigación sobre Políticas Alimentarias.

Soares, F., R. Ribas y G. Hirata (2008), "Achievements and shortfalls of conditional cash transfers: impact evaluation of Paraguay's Tekoporã Programmeme", IPC Evaluation Note, $\mathrm{N}^{\circ} 3$, Brasilia, Centro Internacional de la Pobreza.

Soares, F., R. Ribas y R. Osório (2007), "Evaluating the impact of Brazil's Bolsa Família: cash transfer programmemes in comparative perspective", IPC Evaluation Note, $\mathrm{N}^{\circ} 1$, Brasilia, Centro Internacional de la Pobreza.

Tavares, P. (2008), "Efeito do Programmea Bolsa Família sobre a oferta de trabalho das mães", Anais do XXXVI Encontro Nacional de Economia, Río de Janeiro, Asociación Nacional de Centros de Posgrado en Economía (ANPEC).

Teixeira, C. (2010), "A heterogeneity analysis of the Bolsa Família Programmeme effect on men and women's work supply", Working Paper, $\mathrm{N}^{\circ}$ 61, Brasilia, Centro Internacional de Políticas para el Crecimiento Inclusivo. 